\title{
Global deletion of Optineurin results in altered type I IFN signaling and abnormal bone remodeling in a model of Paget's disease
}

\author{
Sing-Wai Wong $\mathbb{D}^{1,2} \cdot$ Bo-Wen Huang ${ }^{3} \cdot$ Xiangxiang $\mathrm{Hu}^{2} \cdot$ Eui Ho Kim ${ }^{1} \cdot$ Joseph P. Kolb $^{1} \cdot \mathrm{Ricardo}^{\mathrm{J} .}$ Padilla ${ }^{4}$

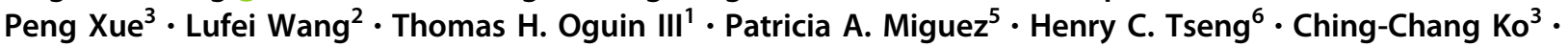 \\ Jennifer Martinez ${ }^{1}$
}

Received: 13 September 2018 / Revised: 29 March 2019 / Accepted: 15 April 2019 / Published online: 10 May 2019

(c) ADMC Associazione Differenziamento e Morte Cellulare 2019

\begin{abstract}
Genome-wide association studies (GWAS) have identified Optineurin (OPTN) as genetically linked to Paget's disease of the bone (PDB), a chronic debilitating bone remodeling disorder characterized by localized areas of increased bone resorption and abnormal bone remodeling. However, only $\sim 10 \%$ of mouse models with a mutation in Optn develop PDB, thus hindering the mechanistic understanding of the OPTN-PDB axis. Here, we reveal that $100 \%$ of aged Optn global knockout $\left(\mathrm{Optn}^{-1-}\right)$ mice recapitulate the key clinical features observed in PDB patients, including polyostotic osteolytic lesions, mixed-phase lesions, and increased serum levels of alkaline phosphatase (ALP). Differentiation of primary osteoclasts ex vivo revealed that the absence of Optn resulted in an increased osteoclastogenesis. Mechanistically, Optn-deficient osteoclasts displayed a significantly decreased type I interferon (IFN) signature, resulting from both defective production of IFN $\beta$ and impaired signaling via the IFN $\alpha / \beta R$, which acts as a negative feedback loop for osteoclastogenesis and survival. These data highlight the dual roles of OPTN in the type I IFN response to restrain osteoclast activation and bone resorption, offering a novel therapeutic target for PDB. Therefore, our study describes a novel and essential mouse model for PDB and define a key role for OPTN in osteoclast differentiation.
\end{abstract}

These authors contributed equally: Henry C. Tseng, Ching-Chang Ko, Jennifer Martinez

Edited by R.A. Knight

Supplementary information The online version of this article (https:// doi.org/10.1038/s41418-019-0341-6) contains supplementary material, which is available to authorized users.

Jennifer Martinez

jennifer.martinez3@nih.gov

1 Immunity, Inflammation, and Disease Laboratory, National Institute of Environmental Health Sciences, National Institutes of Health, Research Triangle Park, NC 27709, USA

2 Oral and Craniofacial Biomedicine Curriculum, School of Dentistry, University of North Carolina at Chapel Hill, Chapel Hill, NC 27599, USA

3 Department of Orthodontics, School of Dentistry, University of North Carolina, Chapel Hill, NC, USA

\section{Introduction}

Paget's disease of the bone (PDB) is an age-dependent bone disease, wherein patients exhibit symptoms of focal areas of bone fragility, bone deformity, pathological fracture, and nerve root compression. PDB pathology has been attributed to osteoclast hyperactivation, leading to excessive bone resorption and irregular bone remodeling

4 Department of Diagnostic Sciences, School of Dentistry, University of North Carolina at Chapel Hill, Chapel Hill, NC 27599, USA

5 Department of Restorative Dentistry, Oral and Craniofacial Health Sciences, School of Dentistry, University of North Carolina at Chapel Hill, Chapel Hill, NC 27599, USA

6 Duke Eye Center and Department of Ophthalmology, Duke University Medical Center, Durham, NC 27710, USA 
[1]. Compared with normal osteoclasts, Pagetic osteoclasts are increased in number, nuclei, activity, and function [2,3]. Furthermore, Pagetic osteoclast precursors display heightened osteoclastogenic potential, as they have an increased responsiveness to RANKL, a critical cytokine for osteoclast differentiation [4]. The molecular mechanisms that underlie hyperactivation of Pagetic osteoclasts, however, remain unknown.

While environmental factors, such as viral infections and toxins, can contribute to the etiology of PDB, recent evidence indicates that genetic factors play a predominant role in PDB pathogenesis [5, 6]. Mutations in Sqstm1, which encodes the autophagy receptor p62, have been repeatedly reported among familial PDB patients [7], and p62 dysfunction results in increased generation and activity of osteoclasts in vitro [8]. Recent genome-wide association studies (GWAS) have identified another autophagy receptor, Optineurin $(O P T N)$, that is genetically linked to PDB [9], with the PDB-associated OPTN variant resulting in a decreased gene expression [10]. OPTN is ubiquitously expressed and mediates the delivery of ubiquitinated cargos, such as damaged organelles, protein aggregates, and intracellular pathogens, to autophagosomes during selective autophagy [11]. OPTN also functions in multiple non-autophagic processes, including vesicle trafficking, Golgi organization, and modulation of the NF-kB pathway [12-16]. In addition to PDB, genetic mutations in OPTN underlie primary openangle glaucoma (POAG) and amyotrophic lateral sclerosis (ALS). While the impacts of OPTN in POAG and ALS have been intensively studied [17-19], the specific role of OPTN in PDB remains to be established.

A recent study demonstrated that while siRNAmediated knockdown of Optn increased osteoclastogenesis in vitro, only $\sim 10 \%$ of 15 - to 18 -month-old mice harboring a loss-of-function mutation in the ubiquitinbinding domain $\left(O p t n^{D 477 N / D 477 N}\right)$ developed PDB lesions [10]. However, the role of OPTN in molecular pathways beyond ubiquitin binding has not be examined in relation to PDB pathogenesis. Therefore, we generated Optn global knockout $\left(\mathrm{Optn}^{--}\right)$mice and performed detailed bone phenotyping analysis. We discovered that $\mathrm{Optn}^{-/}$mice spontaneously develop late-onset polyostotic osteolytic lesions that are reminiscent of clinical findings in PDB patients. Similarly, we observed an increased capacity for osteoclast differentiation with elevated c-Fos expression in the absence of Optn ex vivo. Mechanistically, OPTN's inhibition of osteoclastogenesis is twofold. $\mathrm{Optn}^{-/-}$ osteoclasts produce significantly decreased levels of IFN $\beta$, a known negative regulator of c-Fos [10], as well as display a previously undescribed defect in signaling through the IFN $\alpha / \beta$ R. This defective IFN $\alpha / \beta$ R signaling also resulted in decreased cell death and hence increased survival. Thus, we have generated and characterized a novel and clinically relevant mouse model of PDB and identified a novel OPTN-type I IFN axis in PDB pathogenesis.

\section{Materials and methods}

\section{Animals}

$O p t n^{f l o x f l o x}$ mice on a C57BL/6 background were generated using a targeting vector-inserted LoxP site that flanks the first coding exon and a neomycin selection cassette (Fig. S1). We crossed Optn ${ }^{\text {flox/flox }}$ mice with CMV-Cre mice (Jackson Laboratories, Bar Harbor, ME, USA) to generate $C M V$-Cre; $O p t n^{f l o x}$ mit mice, which were used as breeding pairs. Global Optn knockout $\left(C M V-C r e\right.$; Optn $\left.{ }^{f l o x} f f l o x\right)$ mice are hereafter referred to as $\mathrm{Optn}^{-/-}$mice, and the primers used for genotyping are listed below.

\begin{tabular}{lll}
\hline Primer set & Forward & Reverse \\
\hline A-B3 & taggacctgttaccatgtccca & cectgttcattcaggcccaaag \\
A-D1 & taggacctgttaccatgtccca & cttggctggacgtaaactcctc \\
G2-H2 & gcceggtaccatcaagtcta & aacacctctccagtgcaacc \\
\hline
\end{tabular}

Female $\mathrm{Optn}^{-1-}$ mice were aged for up to 22 months, as aged male mice are more susceptible to spontaneous osteoarthritis, to minimize the influence of age-related osteoarthritis on the phenotype [20, 21]. Ifnar ${ }^{-1-}$ mice were a generous gift from Dr. Michael Fessler (NIEHS). All animal procedures were approved by Institutional Animal Care and Use Committees at the University of North Carolina, Duke University, and NIEHS.

\section{Micro-CT scanning and dual-energy X-ray absorptiometry (DEXA)}

Bones were harvested, fixed in $4 \%$ paraformaldehyde, and scanned by MicroCT Scanco 40 (Scanco Medical, Bassersdorf, Switzerland) in $10-\mu \mathrm{m}$ resolution $(\mathrm{E}=70 \mathrm{kVa}$; $\mathrm{I}=145 \mu \mathrm{A})$. Regions of interest of the cortical and trabecular bone were measured $0.7 \mathrm{~mm}$ proximal to the distal tibiofubular junction, and $0.7 \mathrm{~mm}$ distal to metaphysics of tibia, respectively. The reconstructed solid 3D images were used for visualizing bone morphology and microarchitecture. We measured bone mineral content (BMC) and bone mineral density (BMD) of lumbar spines and femurs by DEXA using LUNAR PIXImus bone densitometer (GE Healthcare, Fairfield, CT, USA). 


\section{Slide staining}

Bones were decalcified in 10\% EDTA for 3 weeks and then processed, paraffin embedded, and sectioned at thickness of $5 \mu \mathrm{m}$. Sections were stained with hematoxylin and eosin (H\&E) for general histology, and with tartrate-resistant acid phosphatase (TRAP) activity to detect osteoclasts, with safranin $\mathrm{O}$ for cartilage. For immunohistochemistry staining, enzymatic antigen retrieval was performed on decalcified sections. After overnight incubation with the primary antibody, sections were incubated with the biotinylated secondary antibody (1:1000; Vector Laboratories, Burlingame, CA, USA). The sections were then immersed in a solution containing avidin-biotin peroxidase complex (Vector Laboratories, Burlingame, CA, USA), and 3,3'diaminobenzidine was used as the chromogen.

\section{Serum measurement of PDB biomarkers}

Sera were isolated from whole blood by centrifugation of $3000 \mathrm{rpm}$ at $4{ }^{\circ} \mathrm{C}$ for $15 \mathrm{~min}$. Serum ALP level was measured by ALP Assay Kit (Abcam, Cambridge, UK). Serum concentrations of IL- 6 was measured by Milliplex Multiplex Assay (EMD Millipore, Burlington, MA, USA). The C-terminal propeptide (sCTx) was measured using ELISA kits (Immunodiagnostic Systems, Boldon, UK).

\section{Osteoclast differentiation and resorptive assay in vitro}

After euthanasia by $\mathrm{CO}_{2}$, tibias and femurs were harvested from 8- to12-week-old mice. Bone marrow cells were flushed into the phenol-free $\alpha$-MEM medium, supplemented with $10 \%$ FBS, L-glutamine, nonessential amino acids, and penicillin/streptomycin. Non-adherent cells were harvested after $24 \mathrm{~h}$ and re-plated at a density of $1.5 \times 10^{5}$ cells $/ \mathrm{cm}^{2}$ with $30 \mathrm{ng} / \mathrm{mL}$ M-CSF (R\&D Systems, Minneapolis, MN, USA). After 2 days, the medium was replenished with $30 \mathrm{ng} / \mathrm{mL}$ M-CSF and $10 \mathrm{ng} / \mathrm{mL}$ RANKL (R\&D Systems Minneapolis, MN, USA) for osteoclast differentiation. After 3 days of culture, cells were fixed and stained with tartrateresistant acid phosphatase (TRAP) to detect osteoclasts [22]. Equal numbers of bone marrow osteoclast precursors were plated and differentiated on Osteo Assay Surface plates (Corning Lifesciences, Tewksburg, MA, USA) for resorption assay. At day 5, the plate was bleached, and areas of resorption pits were quantified using NIH ImageJ.

\section{In vitro IFN $\beta$ and anti-IFNaR antibody treatment}

Bone marrow osteoclast precursors were treated with $1 \mathrm{U}$ IFN $\beta$ (R\&D Systems, Minneapolis, MN, USA) in the osteoclastogenic medium (30 ng/mL M-CSF and $10 \mathrm{ng} / \mathrm{mL}$
RANKL) for 3 days. Similarly, bone marrow osteoclast precursors were treated with an anti-IFNAR antibody or control antibody with a working concentration of $5 \mu \mathrm{g}$ (Leinco, Fenton, MO, USA) in the osteoclastogenic medium for 3 days. Cells were fixed and stained for TRAP activity to evaluate osteoclastogenesis.

\section{Antibodies and western blot analysis}

The following antibodies were from Cell Signaling (Beverly, MA, USA): c-Fos (Cat. \#4384), LC3B (Cat. \#2775), p62 (Cat. \#5114), ATG5 (Cat. \#12994), ATG7 (Cat. \#2631), p65 (Cat. \#8242), phos-p65 (Cat. \#3033), p100/p52 (Cat. \#4882), IкB $\alpha$ (Cat. \#4814), phos-p38 (Cat. \#9215), p38 (Cat. \#9212), phos-ERK1/2 (Cat. \#4370), ERK1/2 (Cat. \#4695), phos-JNK (Cat. \#4668), JNK (Cat. \#9252), phosCREB (Cat. \#9198), CREB (Cat. \#4820), phos-STAT1 (Cat. \#9167), STAT1 (Cat. \#9172), STAT2 (Cat. \#72604), SOCS3 (Cat. \#2932), phos-TBK1 (Cat. \#5483), TBK1 (Cat. \#3504), phos-IRF3 (Cat. \#4947), IRF3 (Cat. \#4302), and RIPK1 (Cat. \#3493). The following antibodies were from Santa Cruz Biotechnology Inc. (Dallas, TX, USA): NFATc1 (Cat. \#sc-7294), OPTN (Cat. \#sc-166576), and actin (Cat. \#sc-1616). Anti-OPTN antibodies were from ProteinTech (Rosemont, IL, USA; Cat. \#10837-1-AP) and Cayman Chemical (Ann Arbor, MI, USA; Cat. \#100000). The myosin VI antibody was from Proteus (Ramona, CA, USA; Cat. \#25-6791); Huntingtin antibody was from Millipore (Burlington, MA, USA; Cat. \#MAB2166); Rab8a antibody was from BD Biosciences (San Jose, CA, USA; Cat. \#610845); phos-STAT2 antibody was from Abcam (Cambridge, MA, USA; Cat. \#ab53132). Secondary antibodies were from Jackson Immuno-Research (West Grove, PA, USA).

Proteins were harvested, and protein concentration was measured as previously described [23]. In total, 5-10 $\mu \mathrm{g}$ of the total protein lysate was resolved by Criterion TGX precast gel (Biorad, Hercules, CA, USA) and transferred to the nitrocellulose membrane using the Trans-Blot Turbo Transfer System (Biorad, Hercules, CA, USA) and immunodetected using appropriate primary and peroxidasecoupled secondary antibodies (Jackson Immunoresearch, West Grove, PA, USA). Proteins were visualized by enhanced chemiluminescence (ECL, Amersham Bioscience, Little Chalfont, UK).

\section{Osteoblast differentiation in vitro}

MC3T3-E1 pre-osteoblasts (Subclone 14, CRL-2594) were obtained from ATCC (Manassas, VA, USA). The cells were seeded at $2 \times 10^{5}$ cells per $35-\mathrm{mm}$ dish, expanded in growth media ( $\alpha$-MEM containing 10\% fetal bovine serum (FBS) and $1 \%$ penicillin/streptomycin), and differentiated with 
growth media supplemented with $10 \mathrm{mM} \quad \beta$ glycerophosphate and $0.2 \mathrm{mmol} / \mathrm{L}$ ascorbic acid. The media was changed every 3 days. After 7, 14, and 21 days, the cells were fixed with $75 \%$ ethanol for $30 \mathrm{~min}$ and then stained with 1\% Alizarin Red (Acros Organics, Geel, Belgium) solution ( $\mathrm{pH}$ 4.2) for $10 \mathrm{~min}$ at RT.

\section{Fluorochrome labeling for in vivo bone formation}

Two fluorochromes (Sigma-Aldrich, St. Louis, MO, USA), calcein $(20 \mathrm{mg} / \mathrm{kg})$ and alizarin red $(30 \mathrm{mg} / \mathrm{kg})$, were used to label in vivo bone formation by intraperitoneal injection. The calcein and alizarin red were administered 5 days and 2 days prior to euthanasia, respectively. Mineral apposition rate (MAR, $\mu \mathrm{m} /$ day), was calculated as the distance between two sequential labels divided by the interlabeling period (days).

\section{RNA isolation and qPCR}

The total RNA was isolated using the RNeasy plus mini kit (Qiagen, Hilden, Germany) and was reverse-transcribed using the iScript DNA synthesis kit (Biorad, Hercules, CA, USA). qPCR was performed using Taqman Universal PCR master Mix (ThermoFisher, Waltham, MA, USA) with Taqman probes (Gapdh: Cat. \#4331182, Ifnbl: Cat. \#4331182). The transcript level of Ifnbl was normalized to the level of Gapdh within each sample using the $\Delta \Delta \mathrm{Ct}$ method.

\section{Microarray}

RNA was isolated from day $2 \mathrm{Optn}^{+/+}$and $\mathrm{Optn}^{-/-}$bonemarrow-derived osteoclasts as described above, and gene expression analysis was conducted using Affymetrix Mouse Genome 4302.0 GeneChip arrays (Affymetrix, Santa Clara, CA, USA). Arrays were scanned in an Affymetrix Scanner 3000 and preliminary analyses were performed with OmicSoft Array Studio (Version 9.0) software.

\section{Flow cytometry}

To assess cell death, osteoclast precursors were harvested 24 and $48 \mathrm{~h}$ after treatment with RANKL in the presence or absence of IFN $\beta$ and stained with Annexin V (1:50), Zombie-Red (1:1000), and CD45 (1:200), as previously described [24]. All antibodies were from Biolegend (San Diego, CA, USA).

\section{Statistical analysis}

For all in vitro studies, three independent experiments were performed. Data are presented as mean \pm SD. Student's $T$ test or analysis of variance (ANOVA) was used to determine the differences among groups. A $p$-value less than 0.05 is considered statistically significant.

\section{Results}

\section{Optineurin is highly expressed in the bone marrow and is upregulated during in vitro osteoclastogenesis}

To explore the relevance of OPTN in bone biology, we first examined its expression pattern in the long bone by immunohistochemistry. OPTN was most highly expressed in the bone marrow (BM), but was also expressed in osteocytes and chondrocytes (Fig. S1A). We next examined the temporal expression of OPTN during in vitro osteoclastogenesis, using both bone marrow cells (Fig. S1B) and RAW 264.7 cells (Fig. S1C) as osteoclast precursors. Osteoclasts were fully differentiated on days 3 and 4 post RANKL stimulation of BM cells and RAW 264.7 cells, respectively, as determined by tartrate-resistant acid phosphatase (TRAP) staining. Expression of OPTN increased during osteoclast differentiation, peaking at days 3 and 2 post RANKL stimulation in BM cells and RAW 264.7 cells, respectively (Fig. S1B-C). Immunofluorescence staining further demonstrated that OPTN was localized in the cytoplasm and perinuclear space of differentiating osteoclasts (Fig. S1B-C).

\section{Young $\mathrm{Optn}^{-1-}$ mice do not display gross skeletal defects in vivo}

To study the role of Optn during bone homeostasis in vivo, we generated CMV-Cre:Optn floxlflox (Optn global knockout mice), hereafter referred to Optn ${ }^{-/-}$mice, (Fig. S2A-B). Western blot analysis confirmed that OPTN was dramatically decreased in multiple organs of $\mathrm{Optn}^{-/-}$mice, confirming global deficiency of this protein (Fig. S2C). At 3 months of age, $\mathrm{Optn}^{-1-}$ mice were phenotypically normal and did not have gross anatomical abnormalities or body size differences compared with $\mathrm{Optn}^{+/+}$littermates (Fig. S3A). Furthermore, skeletal phenotyping using microcomputerized tomography $(\mu \mathrm{CT})$ scanning revealed that 3month-old $\mathrm{Optn}^{-1-}$ mice did not display overt PDB-like lesions in the long bones (Fig. S3B). Young $\mathrm{Optn}^{-/-}$mice also had normal bone morphometric parameters of trabecular and cortical bones compared with $\mathrm{Optn}^{+/+}$littermates (Fig. S3C). Similarly, serum levels of alkaline phosphatase (ALP) activity and the C-terminal telopeptide (CTX-1), both biomarkers indicative of bone turnover, were not significantly elevated between $\mathrm{Optn}^{+/+}$and $\mathrm{Optn}^{-/-}$mice at 3 months of age (Fig. S3D). 
Table 1 Phenotypic analysis of skeletal abnormalities in 3-, 8-, 12-, 16-, and 22-month old $\mathrm{Optn}^{+/+}$and $\mathrm{Optn}^{-/-}$mice.

\begin{tabular}{|c|c|c|c|c|c|c|}
\hline Genotype & Pathology & 3 months & 8 months & 12 months & 16 months & 22 months \\
\hline \multirow[t]{5}{*}{ Optn $+/+$} & Skeletal abnormalities & $0 \%$ & $0 \%$ & $0 \%$ & $0 \%$ & $0 \%$ \\
\hline & Osteolytic lesion & $0 \%$ & $0 \%$ & $0 \%$ & $0 \%$ & $0 \%$ \\
\hline & Osteoscleorotic-osteolytic lesion & $0 \%$ & $0 \%$ & $0 \%$ & $0 \%$ & $0 \%$ \\
\hline & Spinal cord/nerve root compression & $0 \%$ & $0 \%$ & $0 \%$ & $0 \%$ & $0 \%$ \\
\hline & Facial deformity & $0 \%$ & $0 \%$ & $0 \%$ & $0 \%$ & $0 \%$ \\
\hline \multirow[t]{5}{*}{$\mathrm{Optn}^{-/-}$} & Skeletal abnormalities & $0 \%$ & $0 \%$ & $0 \%$ & $50 \%$ & $100 \%$ \\
\hline & Osteolytic lesion & $0 \%$ & $0 \%$ & $0 \%$ & $50 \%$ & $100 \%$ \\
\hline & Osteoscleorotic-osteolytic lesion & $0 \%$ & $0 \%$ & $0 \%$ & $0 \%$ & $40 \%$ \\
\hline & Spinal cord/nerve root compression & $0 \%$ & $0 \%$ & $0 \%$ & $0 \%$ & $40 \%$ \\
\hline & Facial deformity & $0 \%$ & $0 \%$ & $0 \%$ & $0 \%$ & $20 \%$ \\
\hline
\end{tabular}

\section{Optineurin deficiency in vivo results in PDB-like lesions in aged mice}

We next performed skeletal phenotyping of the mice at 8 , 12, and 16 months of age. Similar to 3-month-old mice, neither genotype displayed any skeletal abnormalities or lesions at 8 or 12 months of age (Fig. S4A-B, Table 1). At 16 months of age, however, $50 \%$ of $\mathrm{Optn}^{-1-}$ mice had developed incipient, monostotic, localized osteolytic lesions in the tibiae (Fig. S4C). While there was no significant difference in serum ALP levels between $\mathrm{Optn}^{+/+}$and Optn $^{-/-}$mice at 8, 12, and 16 months, serum ALP levels had significantly increased in $\mathrm{Optn}^{-1-}$ mice between 8 and 16 months, signaling an age-induced onset of PDB in the absence of Optn (Fig. S4D).

Because PDB primarily affects the elderly population with a peak incidence between 70 and 80 years of age [25], we next performed skeletal phenotyping of 22-month-old mice, which is equivalent to humans at 70 years old [26]. Strikingly, $100 \%$ of aged $\mathrm{Optn}^{-1-}$ mice had developed polyostotic, localized osteolytic lesions in femurs, tibiae (Fig. 1a, b), calvaria (Fig. 1c), lumbar vertebra (Fig. 1d), and fibulas (Fig. 1e), which are the most commonly affected bones of PDB, and these lesions phenotypically resembled the early or osteolytic stage of PDB (Table 1). In addition, $20 \%$ of $\mathrm{Optn}^{-1-}$ mice exhibited facial deformities (Fig. 1c) and $40 \%$ of $\mathrm{Optn}^{-1-}$ mice had bone hypertrophic lesions (Fig. 1e), pathologies associated with the intermediate or osteoblastic stage of PDB (Table 1). Furthermore, spinal cord/nerve root compression (Fig. 1d) and pathological fractures (Fig. 1e) were also seen in the affected bones of aged $\mathrm{Optn}^{-l-}$ mice (Table 1). Aged $\mathrm{Optn}^{-1-}$ mice also contained decreased cortical bone components in unaffected bones (Fig. S4F) and displayed a decrease in bone mineral density (BMD) (Fig. S4G). Taken together, these results demonstrate that Optineurin deficiency results in agedependent, localized bone lesions that are phenotypically consistent with PDB.

\section{Pagetic lesions in Optn ${ }^{-1-}$ animals are characterized by hyperosteoclastogenesis}

The osteolytic lesions observed in $\mathrm{Optn}^{-/-}$mice are characteristic of disproportionate bone turnover, indicating that OPTN exerts its effect by either enhancing bone resorptive activity by osteoclasts or reducing boneformation activity by osteoblasts. Although OPTN is induced during osteogenesis in vitro (Fig. S5A), we did not observe any difference in vivo bone formation as determined by mineral apposition rate (MAR) between young $\mathrm{Optn}^{+/+}$and $\mathrm{Optn}^{-/-}$mice (Fig. S5B). Thus, we hypothesized that osteolytic lesions observed in aged $\mathrm{Optn}^{-1-}$ mice were a result of altered osteoclast activity.

We next performed histological analysis of bones of aged $\mathrm{Optn}^{+/+}$and $\mathrm{Optn}^{-/-}$mice. H\&E and TRAP staining of the distal femur of $\mathrm{Optn}^{-/-}$mice confirmed osteolytic lesions and revealed increased bone resorption, with osteoclast-filled pits within the compact bone of $\mathrm{Optn}^{--}$mice, while bones from age-matched $\mathrm{Optn}^{+/+}$mice lacked these pathological features (Fig. 2a). Cross-sectional analysis of a mixed osteoscleorotic-oste7olytic lesion in the proximal tibia of an $\mathrm{Optn}^{-1-}$ mouse shows that these lesions comprise numerous osteoclasts located within the inner surface of cortical bone and adjacent trabecular surfaces (Fig. 2b, c). In addition, the mixed lesion also displayed secondary endochondrial bone formation (Fig. 2b). Focal lesions in the affected bones from $\mathrm{Optn}^{-1-}$ mice contained significantly more osteoclasts per area examined compared with $\mathrm{Optn}^{+/+}$mice (Fig. 2d), and each $\mathrm{Optn}^{-/-}$osteoclast contained more nuclei than $\mathrm{Optn}^{+/+}$ osteoclasts, indicative of increased osteoclast activity (Fig. 2e).

The gold standard for clinical diagnosis of PDB in human patients is increased serum levels of ALP, in response to increased osteoclastic bone resorption [27]. Similar to PDB patients, we found that aged $\mathrm{Optn}^{-1-}$ mice had significantly increased ALP activity in their sera, compared with age-matched $\mathrm{Optn}^{+/+}$mice, and thus recapitulate the classical diagnostic biomarker for PDB 
A

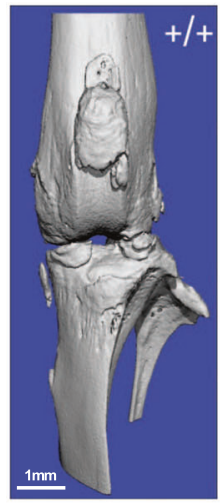

D
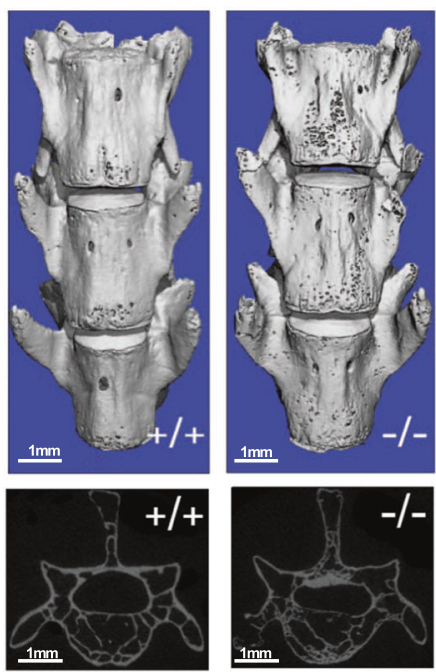

Fig. 1 Aged $\mathrm{Optr}^{-/-}$mice develop Pagetic lesions. a-e Representative micro-CT 3D reconstructed images of osteolytic lesions in the distal femur (a, b), proximal tibia (b), calvaria (c), and lumbar vertebral body (d) of 22-month-old female Optn $^{-/}$mice (-/-), compared with agematched female $\mathrm{Optn}^{+/+}$mice $(+/+)$. Irregular bone remodeling in the calvaria and facial deformity (c) was observed in $\mathrm{Optn}^{-/-}$mice (-/-).

(Fig. 2f). Furthermore, serum levels of CTX-1, the bone resorption biomarker, were significantly increased in aged $\mathrm{Optn}^{-/-}$mice compared with Optn ${ }^{+/+}$mice (Fig. 2f). However, serum levels of IL-6, a cytokine that has been associated with PDB [28], were not affected by loss of OPTN in aged animals (Fig. S5C). Taken together, our data demonstrate that $\mathrm{Optn}^{-1-}$ mice exhibit serum and histological features of PDB associated with increased osteoclastogenesis, and the $\mathrm{Optn}^{-1-}$ mice represent a novel and clinically relevant mouse model for PDB.

\section{OPTN deficiency enhances in vitro osteoclastogenesis}

We next explored the mechanisms by which deficiency of OPTN augments osteoclast formation. $\mathrm{Optn}^{-1-}$ bone
C
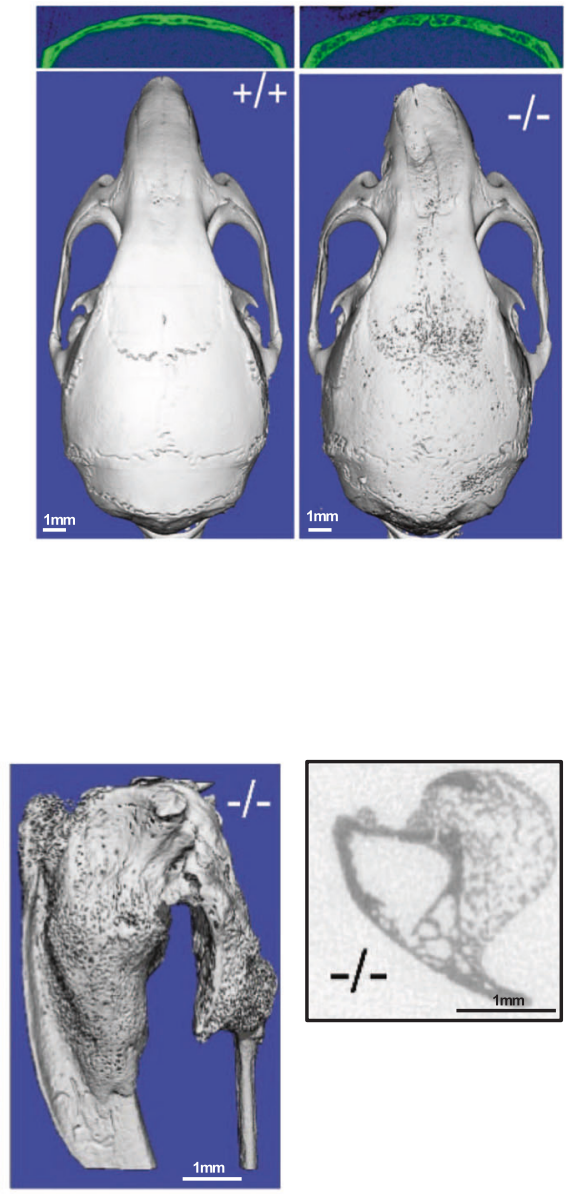

The affected L4 of the lumbar vertebral body displayed a chaotic trabecular structure, which causes spinal stenosis and spinal cord/nerve root compression (d). e Aged $\mathrm{Optn}^{-/-}$mice had mixed-stage lesions in the proximal tibia and fibula, and the affected fibula displayed a pathological fracture. ( $n=5-7$ mice per genotype)

marrow osteoclast precursors generated significantly increased numbers of osteoclasts ex vivo, compared with $\mathrm{Optn}^{+/+}$bone marrow precursors (Fig. 3a). Furthermore, $\mathrm{Optn}^{-1-}$ osteoclasts differentiated ex vivo on calcium phosphate-coated plates displayed increased resorptive activity, as indicated by larger resorption pit areas compared with $\mathrm{Optn}^{+/+}$osteoclasts (Fig. 3b). Finally, Optn ${ }^{-1-}$ precursor cells expressed significantly higher levels of c-Fos and NFATc1, two important osteoclast differentiation factors, post RANKL treatment (Fig. 3c). Collectively, these data demonstrate that OPTN is a key negative regulator of osteoclastogenesis.

As autophagy has been shown to be involved in osteoclast differentiation [29] and OPTN is an autophagy receptor, we first asked if the absence of OPTN alters autophagic induction during osteoclastogenesis. 
A

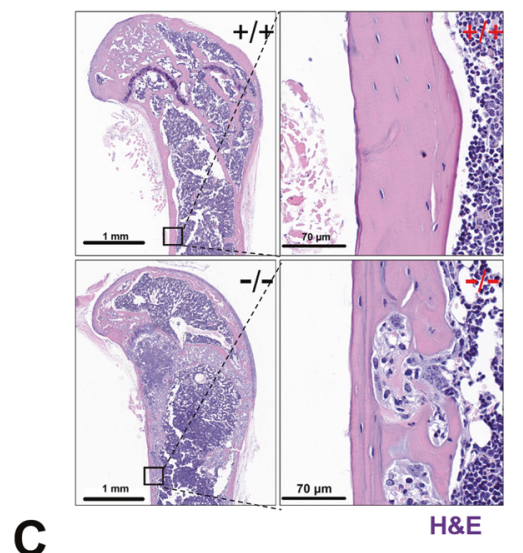

B

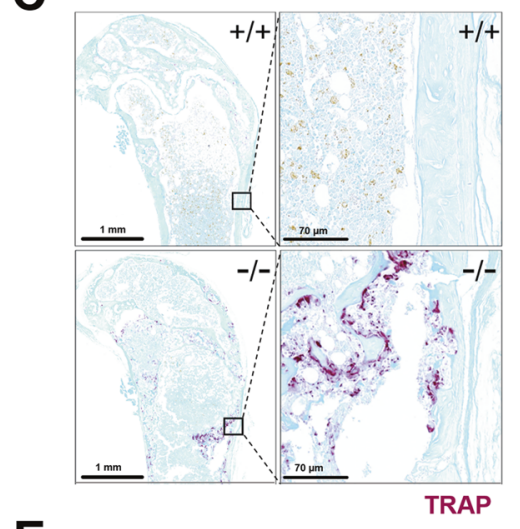

D
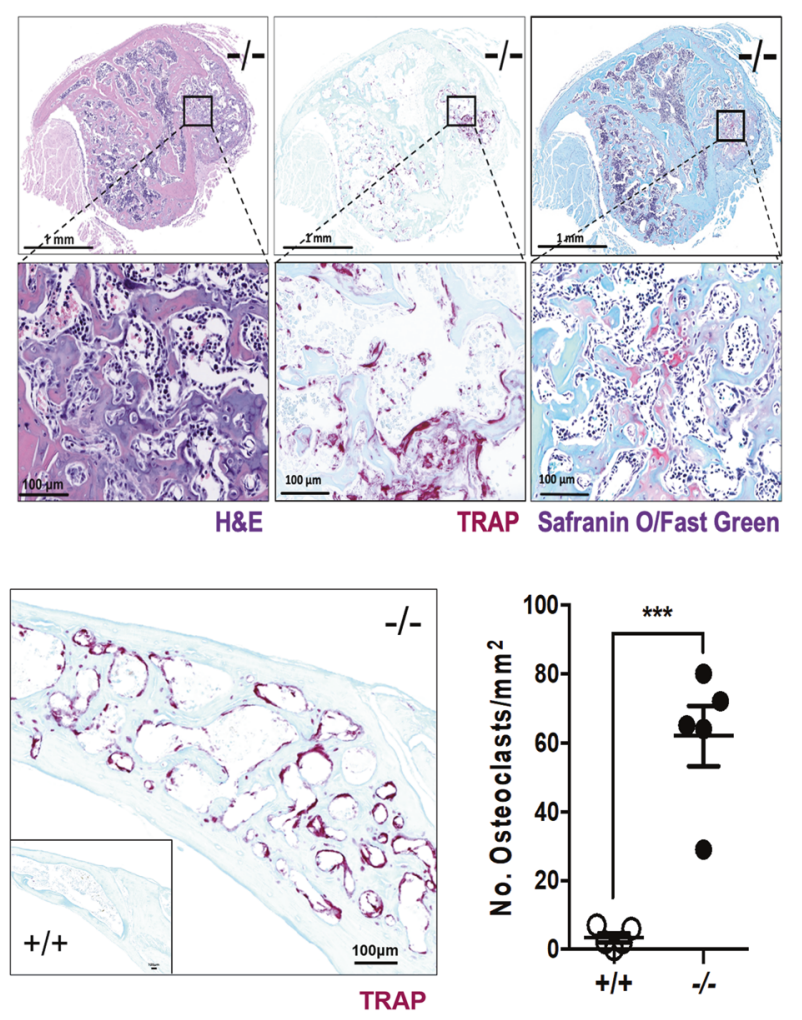

E
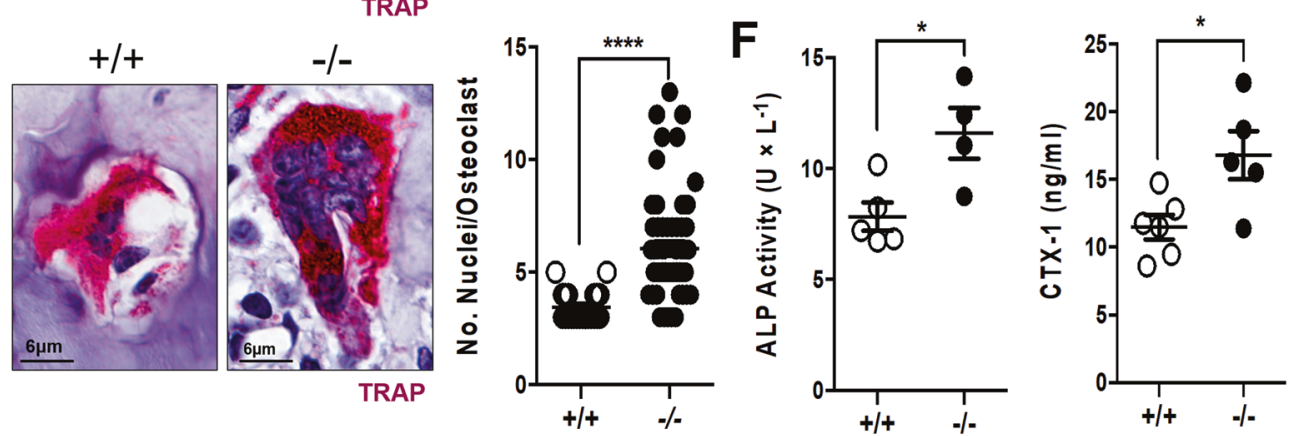

Fig. 2 Increased osteoclastogenesis in aged $\mathrm{Optn}^{-/-}$mice. a H\&E staining of the distal femur of 22-month-old female $\mathrm{Optn}^{+/+}(+/+)$and $\mathrm{Optn}^{-/-}$ $(-/-)$ mice. b Staining of a mixed osteoscleorotic-osteolytic lesion in the proximal tibia of 22-month-old Optn $^{-/-}$(-/-) mouse with H\&E (left), TRAP (middle), and Safranin O/Fast Green (right). c, d TRAP staining in the distal femur $(\mathbf{c})$ and parietal bone $(\mathbf{d})$ in $22-$-month-old $O p t n^{+/+}(+/+)$ and $\mathrm{Optn}^{-\gamma_{-}}(-/-)$mice. Quantification of osteoclasts per $\mathrm{mm}^{2}$ of unaffected bones of $\mathrm{Optn}^{+/+}(+/+)$and lesion sites of $\mathrm{Optn}^{-{ }_{-}}(-/-)$mice $(* * * p$ $<0.001, n=5$ mice). e Representative images of multi-nucleated osteoclast in the lesion of 22-month-old $\mathrm{Optn}^{+/+}(+/+)$and $\mathrm{Optn}^{-/-}(-/-)$mice. Quantification of the number of nuclei per osteoclast in 22-month-old $O_{p t n^{+/+}}(+/+)$and Optn $^{-/-}(-/-)$mice $(* * * * p<0.0001, n=60$ osteoclasts $)$. f Serum levels of alkaline phosphatase (ALP) and collagen type I C-telopeptide (CTX-1) in 22-month-old Optn $^{+/+}(+/+)$and Optn $^{-/-}(-/-)$mice $(* p<0.05, * * * p<0.001, n=4-6$ per genotype)

Intriguingly, $\mathrm{Optn}^{-1-}$ osteoclasts had a similar transformation of the autophagy marker LC3-I to LC3-II and degradation of p62, as well as expression of ATG5 and ATG7, compared with $\mathrm{Optn}^{+/+}$osteoclasts, suggesting that autophagy is unaffected in $\mathrm{Optn}^{-1-}$ osteoclasts under osteoclastogenic conditions (Fig. 3d). Activation of the c-FosNFATc1 axis for osteoclastogenesis involves the NF- $\mathrm{BB}$ pathway [30]. As OPTN has been shown to suppress NF-kB activation [12], we examined the phosphorylation of p65, a member of the canonical NF-kB pathway, in $\mathrm{Optn}^{+/+}$and $O$ ptn $^{-1-}$ osteoclast precursors after RANKL treatment. The level of phosphorylated p65 in $\mathrm{Optn}^{-/-}$osteoclast precursors was similar to that in $\mathrm{Optn}^{+/+}$osteoclast precursors at both early time points (Fig. 3e) and late time points (Fig. 3f). Similarly, the degradation of the $\mathrm{I} \mathrm{KB} \alpha$, a negative regulator of canonical NF- $\mathrm{KB}$ activation, was equivalent in $\mathrm{Optn}^{+/+}$and $\mathrm{Optn}^{-/-}$cells after RANKL treatment (Fig. 3e). While previous studies utilized the Optn ${ }^{D 477 N /}$ 
A
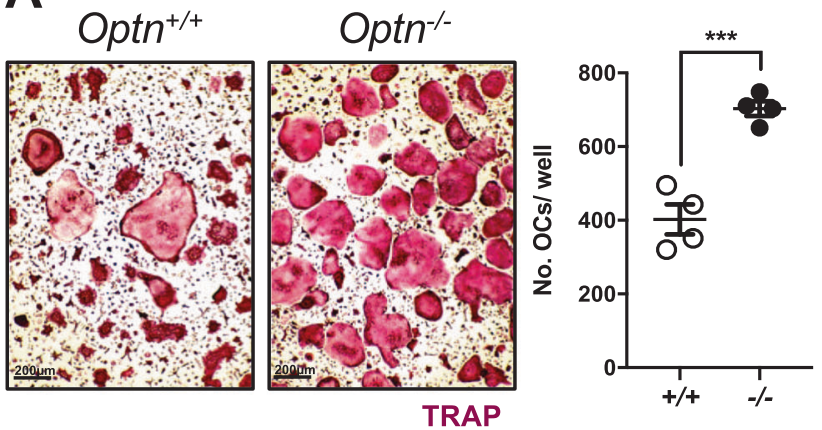

B
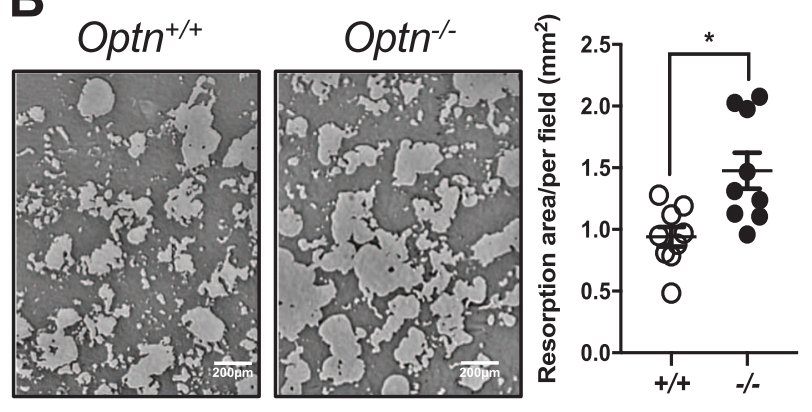

C
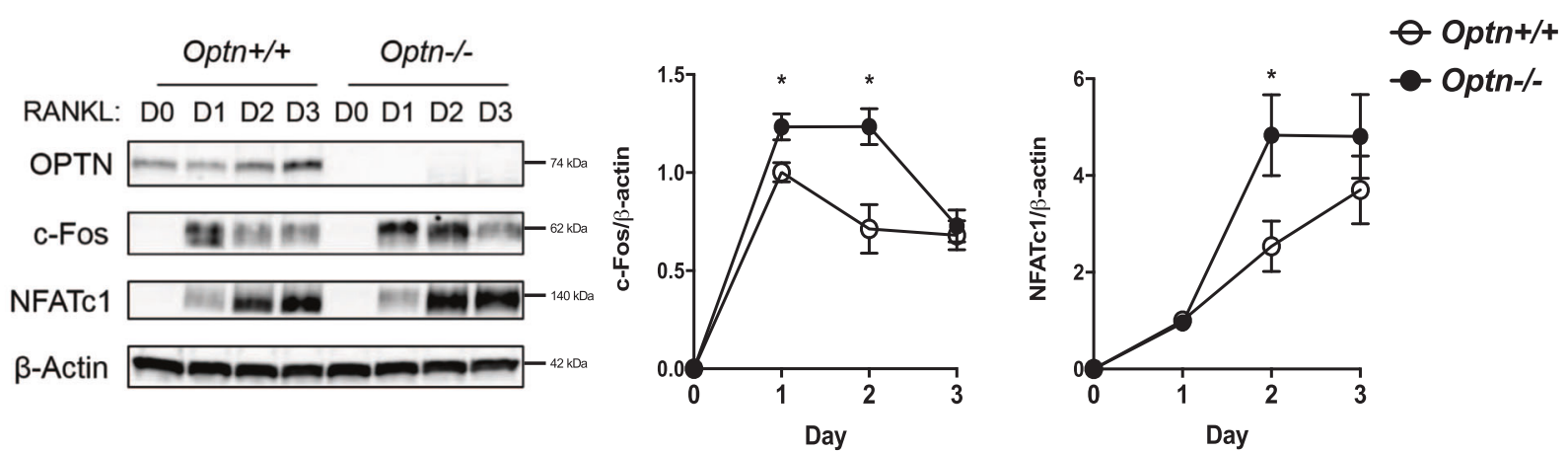

D

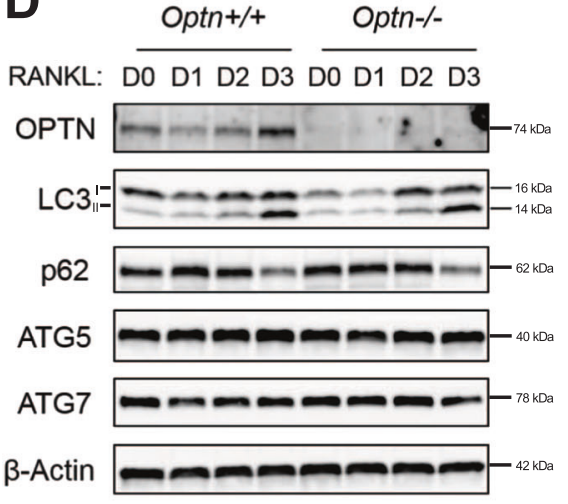

F

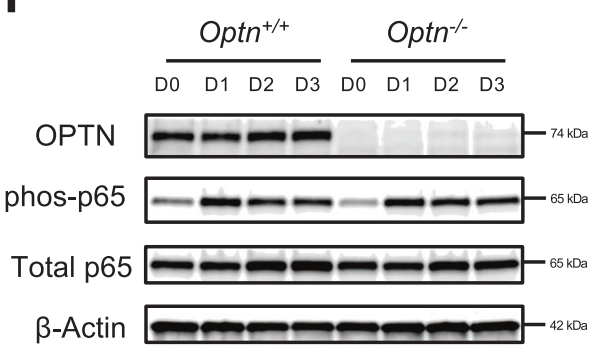

E

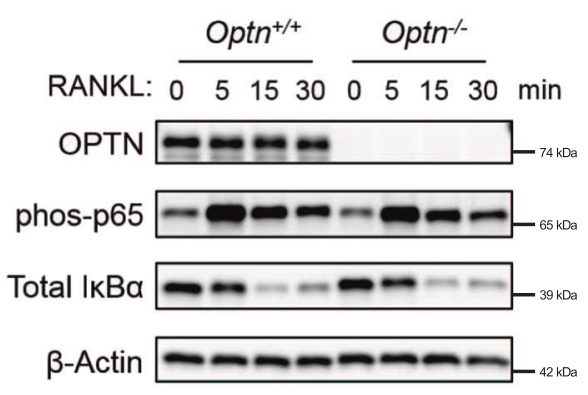

G

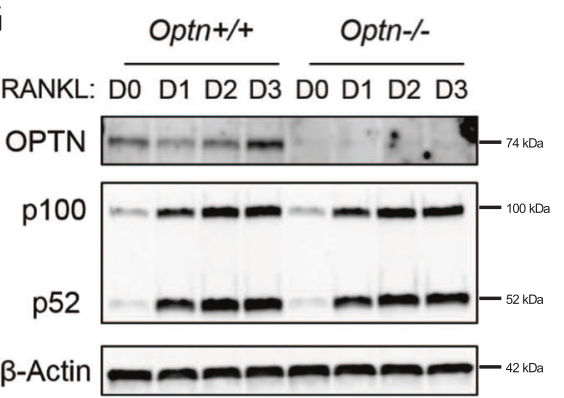

${ }^{D 477 N}$ model, which results in OPTN protein that is unable to bind Ly63-linked ubiquitin chains, but can still interact with other proteins via its other un-mutated domains [10], our constitutive knockout model displayed no differences in $\mathrm{NF}-\kappa \mathrm{B}$ activation during osteoclastogenesis. Collectively, these data indicate that while defects in OPTN's ability to bind ubiquitin can result altered $N F-\kappa B$ activation, the absence of OPTN does not.

In addition, we also assessed noncanonical NF- $\mathrm{B}$ activation by examining the processing of p100 to p52 during 
Fig. 3 OPTN deficiency promotes osteoclastogenesis in vitro. a $\mathrm{Optn}^{+/+}$and $\mathrm{Optn}^{-/-}$bone marrow-derived precursors from 8- to12week-old mice were cultured under osteoclastogenic conditions, and osteoclast (OC) differentiation was assessed by quantification of the number of multi-nucleated TRAP-positive cells on day 3 post RANKL treatment. Representative images are shown. $(* * * p<0.001, n=4$ wells). b $\mathrm{Optn}^{+/+}$and $\mathrm{Optn}^{-1-}$ bone marrow-derived precursors from 8- to 12-week-old mice were cultured under osteoclastogenic conditions on Osteo Assay Surface plates, and resorptive surfaces were quantified on day 5 post RANKL treatment. Representative images are shown. ( ${ }^{*} p<0.05, n=9$ wells). c Western blot of OPTN, c-Fos, and NFATc1 in $\mathrm{Optn}^{+/+}$and $\mathrm{Optn}^{-/-}$OC at days 0-3 of OC differentiation. Densitometry was calculated with ImageJ $(* p<0.05, n=3$ blots). d, g $\mathrm{Optn}^{+/+}$and $\mathrm{Optn}^{-1-}$ bone marrow-derived precursors were cultured under osteoclastogenic conditions, and autophagic activity (d) and noncanonical NF-kB signaling (g) was assessed by Western blot on days 0-3 post-RANKL treatment from the sample protein lysates. In D, cells were probed for OPTN, LC3, p62, ATG5, ATG7, and $\beta$-actin. In G, the Western blots were reprobed for p100/ p52 with the same OPTN and $\beta$-actin bands shown for reference. (e-f) $\mathrm{Optn}^{+/+}$and $\mathrm{Optn}^{-/-}$bone marrow-derived precursors were cultured with RANKL for 0-60 minutes or 0-3 days, to assess the activation of canonical NF-kB signaling by Western blot. Cells were probed for OPTN (e-f), phos-p65 (e-f), Total p65 (f), Total IkB (e), and $\beta$-actin $(\mathbf{e}-\mathbf{f})$

osteoclastogenesis. Optn ${ }^{-1-}$ osteoclast precursors displayed similar levels of the cleaved p52 protein compared with Optn $+/+$ osteoclast precursors (Fig. 3f). These results demonstrate that OPTN regulates osteoclast differentiation independently of autophagy, canonical, or noncanonical NF- $\mathrm{B}$ activation.

We next evaluated other possible signaling pathways implicated in osteoclastogenesis and observed equivalent phosphorylated levels of JNK1/2, ERK1/2, p38, and CREB (Fig. S6A, B) in $\mathrm{Optn}^{+/+}$and $\mathrm{Optn}^{-/-}$osteoclast precursors post RANKL treatment. We further examined the expression of known OPTN-binding partners, RAB8A, Myosin VI, and Huntingtin (HTT) in Optn $^{-1-}$ osteoclasts relative to $\mathrm{Optn}^{+/+}$osteoclasts, and we found that their expression levels were comparable (Fig. S6C). Therefore, OPTN deficiency does not affect classical osteoclastogenic pathways in osteoclast precursors during RANKL treatment.

\section{OPTN is required for both the production of type I IFN and signaling via the IFNa/ $\beta$ R}

To investigate potential transcriptional differences between $\mathrm{Optn}^{+/+}$and $\mathrm{Optn}^{-1-}$ osteoclasts, genome-wide transcriptional profiling was performed at day 3 of osteoclast differentiation ex vivo. Microarray revealed that many critical pathways were upregulated in $\mathrm{Optn}^{-1-}$ osteoclasts, including genes regulating cytoskeletal rearrangements, cell cycle, cytokine and chemokine expression, and osteoclast differentiation and function, compared with $\mathrm{Optn}^{+/+}$osteoclasts (Fig. S6D). Consistent with our protein results, the transcription of Rab8a/b, Myosin VI, and Htt was equivalent between $\mathrm{Optn}^{+/+}$and $\mathrm{Optn}^{-/-}$osteoclasts (Fig. S6D). In addition, there was no difference in transcription of other components known to interact with OPTN, such as Ripk1, Cyld, Sqstm1, and Hacel, between $\mathrm{Optn}^{+/+}$and $\mathrm{Optn}^{-/-}$ osteoclasts (Fig. S6D).

We did, however, observe a significant difference in the expression of genes associated with the type I IFN signature. Genes such as Ifitm10, Ifitm5, Ifnal, Irf7, and Ifnar2 were dramatically reduced in $O p t n^{-1-}$ osteoclasts compared with $\mathrm{Optn}^{+/+}$osteoclasts (Fig. 4a). Conversely, expression of Socs3, a negative regulator of type I IFN signaling [31], was significantly upregulated in $\mathrm{Optn}^{-1-}$ osteoclasts compared with $\mathrm{Optn}^{+/+}$osteoclasts (Fig. 4a). Engagement of the IFN $\alpha / \beta$ R with type I IFN (typically IFN $\beta$ in myeloidderived cells, such as osteoclasts) results in the phosphorylation of STAT1 [32]. In response to RANKL treatment, $\mathrm{Optn}^{-/-}$osteoclast precursors displayed lower levels of the total and phosphorylated STAT1 and higher levels of SOCS3, suggesting that Optn deficiency confers a reduced activation of the type I IFN response in response to RANKL (Fig. 4b).

We next explored whether this reduced type I IFN signaling in $\mathrm{Optn}^{-/-}$osteoclasts resulted from decreased IFN $\beta$ production or defective IFN $\alpha / \beta \mathrm{R}$ signaling, or both. We first observed that $\mathrm{Optn}^{-/-}$osteoclast precursors generated significantly lower levels of Ifnbl in response to RANKL treatment (Fig. 4c), compared with $\mathrm{Optn}^{+/+}$precursors. RANKL-induced IFN $\beta$ production does not require canonical TBK1 and IRF3 machinery [33], and we indeed observed that RANKL failed to activate IRF3 in osteoclast precursors and induced a comparable level of TBK1 phosphorylation, which is required for $\mathrm{NF}-\kappa \mathrm{B}$ activities, between $\mathrm{Optn}^{+/+}$and $\mathrm{Optn}^{-/-}$precursors (Fig. S6E). Next, we examined signaling downstream of IFN $\beta$ engagement of IFN $\alpha / \beta$ R during IFN $\beta$ treatment. Strikingly, Optn ${ }^{-/-}$ osteoclast precursors displayed defective IFN $\alpha / \beta \mathrm{R}$ signaling in response to IFN $\beta$ stimulation, as evidenced by decreased total and phosphorylated STAT1/2 (Fig. 4d). As expected, IFN $\alpha / \beta R$-deficient (Ifnar $\left.{ }^{-/}\right)$osteoclast precursors displayed a completely abolished type I IFN signaling in response to RANKL or IFN $\beta$ (Fig. S6F-G). Taken together, these results demonstrate that OPTN is critical for both the production of type I IFN and efficient signaling via the IFN $\alpha / \beta$ R pathway.

Type I IFN signaling can also promote RIPK3-mediated necroptosis [24], therefore we next examined if defects in IFN $\beta$ production by $\mathrm{Optn}^{-/-}$osteoclasts conferred a survival advantage compared with $\mathrm{Optn}^{+/+}$osteoclasts. Treatment of $\mathrm{Optn}^{-/-}$osteoclast precursors with RANKL, a member of the TNF superfamily [34], resulted in significantly increased survival at both 24 and $48 \mathrm{~h}$ post RANKL treatment, compared with $\mathrm{Optn}^{+/+}$precursors (Fig. 5a). In order to determine if exogenous IFN $\beta$ treatment 


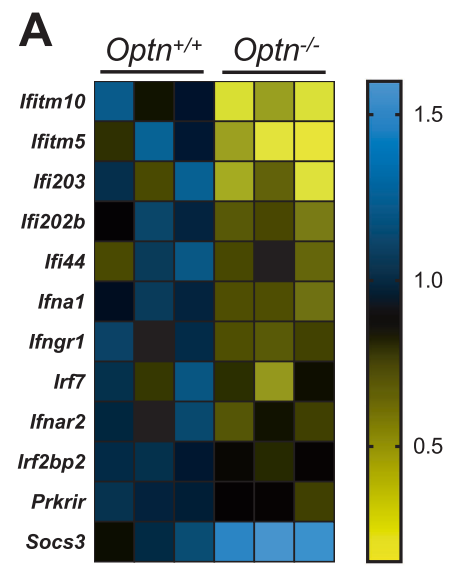

D

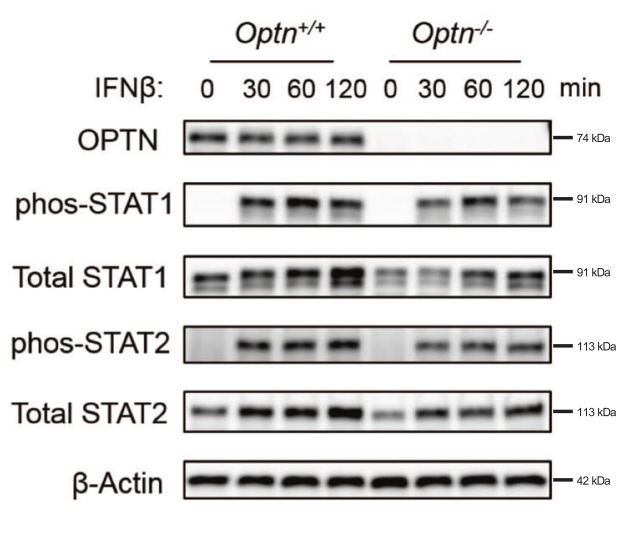

Fig. 4 OPTN deficiency affects RANKL-induced IFN $\beta$ production and type I IFN activation. a Microarray analysis of $\mathrm{Optn}^{+/+}$and $\mathrm{Optn}^{-/-}$ OC on day 2 post RANKL treatment. Heatmap representing colorcoded expression levels of differentially expressed genes in the type I IFN signature ( $\log 2$ values) of biological triplicate samples of $\mathrm{Optn}^{+/+}$ and $\mathrm{Optn}^{-1-}$ OC. b Western blot of OPTN, phos-STAT1, Total STAT1, and SOCS3 expression in $\mathrm{Optn}^{+/+}$and $\mathrm{Optn}^{-1-}$ OC

during osteoclastogenesis could modulate cell death in $\mathrm{Optn}^{-/-}$osteoclast precursors, we differentiated $\mathrm{Optn}^{+/+}$ and $\mathrm{Optn}^{-1-}$ precursors into osteoclasts with RANKL in the absence or presence of IFN $\beta$. The survival advantage observed in $\mathrm{Optn}^{-1-}$ osteoclast precursors was rescued at $48 \mathrm{~h}$ post RANKL/IFN $\beta$ treatment, compared with $\mathrm{Optn}^{+/+}$ precursors (Fig. 5b, S7A).

As IFN $\beta$ is also an established negative regulator of osteoclastogenesis [33], we next asked if exogenous IFN $\beta$ treatment during osteoclastogenesis could rescue the hyperdifferentiation observed in $\mathrm{Optn}^{-/-}$osteoclasts.
C

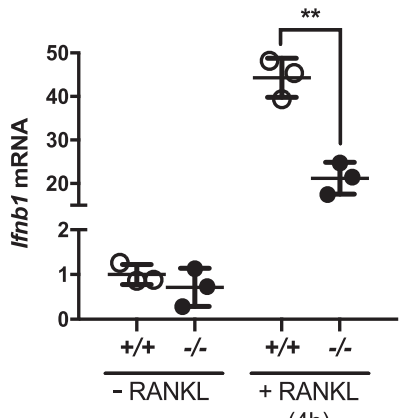

(4h)
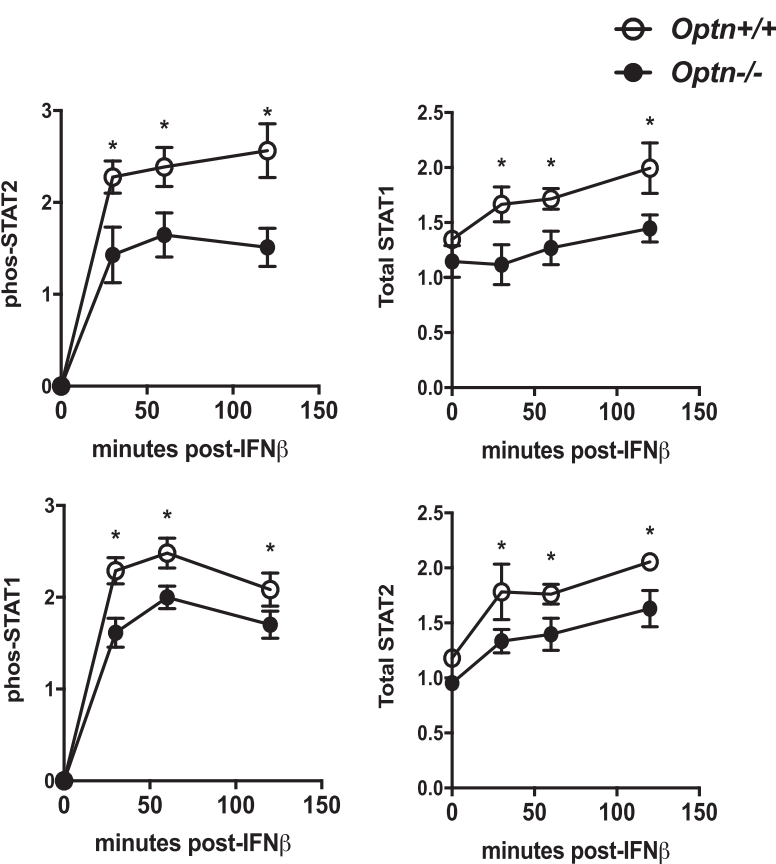

precursors treated with RANKL at 4 and $6 \mathrm{~h}$. c The mRNA level of Ifnbl in OC precursors treated with RANKL at $4 \mathrm{~h}$ was determined using qPCR. ( ${ }^{*} p<0.05, n=3$ wells). d $O p t n^{+/+}$and $O p t n^{-1-}$ OC precursors from 8- to12-week-old mice were treated with IFN $\beta$ and assessed for type I IFN signaling via western blot for phos-STAT1, phos-STAT2, Total STAT1, and Total STAT2. Densitometry was calculated with ImageJ $(* p<0.05, n=3$ blots)

Recombinant IFN $\beta$ was sufficient to inhibit the number of osteoclasts generated by $\mathrm{Optn}^{-/-}$precursors, as evidenced by TRAP staining (Fig. 5c). Furthermore, antibodymediated inhibition of IFN $\alpha / \beta \mathrm{R}$ signaling ( $\alpha$ IFNAR) resulted in a significant increase in osteoclastogenesis of both $\mathrm{Optn}^{+/+}$and $\mathrm{Optn}^{-/-}$precursors (Fig. 5c). Importantly, IFN $\alpha / \beta$ R signaling blockade had a larger effect on $\mathrm{Optn}^{+/+}$ precursors, essentially normalizing the $\mathrm{Optn}^{-1-}$ osteoclastogenic phenotype (Fig. 5d). This suggests that the excess OC differentiation of $\mathrm{Optn}^{-/-}$cells is mediated by a deficit in native IFN signaling. 

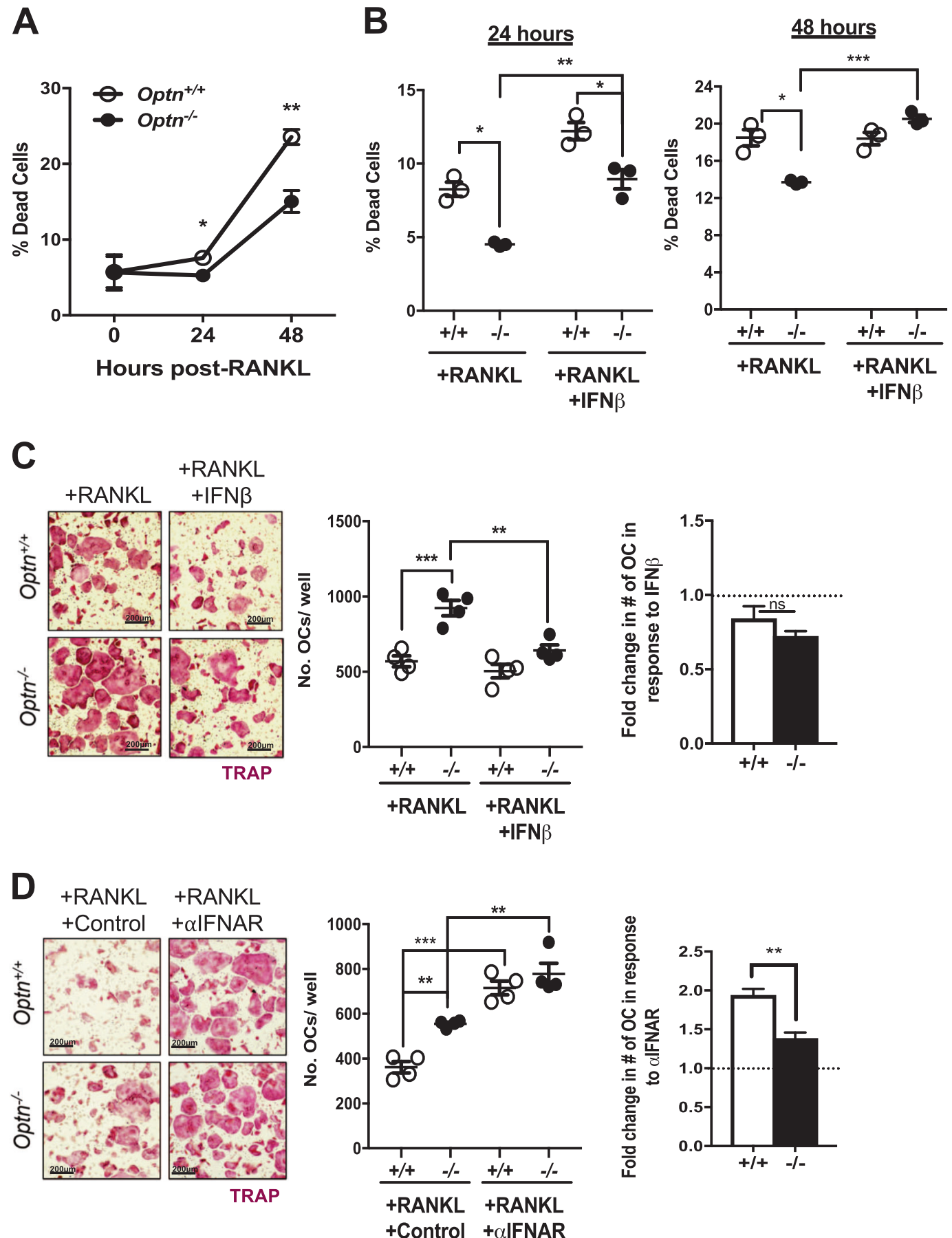

Fig. 5 Recombinant IFN $\beta$ can rescue hyperosteoclastogenesis observed during OPTN deficiency. a $\mathrm{Optn}^{+/+}$and $\mathrm{Optn}^{-1-}$ OC precursors from 8- to 12-week-old mice treated with RANKL, and cells were harvested at 0,24 , and $48 \mathrm{~h}$ post RANKL treatment and analyzed by flow cytometry for cell death by Annexin V and Zombie-Red. \% Dead cells calculated as percentage Annexin $\mathrm{V}^{+}$Zombie-Red $^{+}$of $\mathrm{CD} 45^{+}$cells $(* p<0.05, * * p<0.01, n=6$ wells $) . \quad$ b $O p t n^{+/+}$and $\mathrm{Optn}^{-1-}$ OC precursors treated with RANKL with or without IFN $\beta$, and cells were harvested at 24 and $48 \mathrm{~h}$ post RANKL/ IFN $\beta$ treatment

Collectively, we have described the $\mathrm{Optn}^{-1-}$ mouse as a novel and clinically relevant model for PDB in vivo and have demonstrated that OPTN is required to maintain and analyzed by flow cytometry for cell death by Annexin V and Zombie-Red. \%Dead cells calculated as percentage Annexin $\mathrm{V}^{+}$ Zombie-Red ${ }^{+}$of CD $45^{+}$cells $\left({ }^{*} p<0.05,{ }^{* * *} p<0.001, n=3\right.$ wells). c TRAP staining of $\mathrm{Optn}^{+/+}$and $\mathrm{Optn}^{-1-}$ osteoclast precursors treated with RANKL with or without IFN $\beta$ for 3 days to assess OC formation (** $p<0.01, n=4$ wells). h TRAP staining of osteoclast precursors under osteoclastogenic conditions with control or IFN $\alpha / \beta R$ blocking ( $\alpha$ IFNAR) antibody for 3 days $(* p<0.05, * p<0.01$, *** $p<0.001$, $n=4$ wells)

homeostatic levels of osteoclastogenesis in vivo and ex vivo, the absence of which results in hyperactive osteoclast differentiation and activity. In addition, we 
demonstrate that OPTN functions on two levels to regulate osteoclast differentiation-the production of type I IFN and signaling of type I IFN through the IFN $\alpha / \beta R$ (Fig. 6), and the net effect of this defect is increased osteoclastogenesis, as well as defects in cell death, opening the door for possible interferon intervention for the treatment of PDB.

\section{Discussion}

Recent advances in the underlying genetic influences on the onset and severity of PDB have shed light onto the root causes of this disease and opened up avenues for deeper biological understanding. Here, we performed extensive in vivo skeleton characterization of the Optn-deficient mouse, which exhibits full penetrance and multiple key clinical manifestations of PDB in an age-dependent manner, in contrast to the Optn ${ }^{D 477 N / D 477 N}$ mutant mice, in which only $~ 10 \%$ of $15-18$-month-old mice develop PDB lesions [10, 35]. Therefore, this Optn-deficient mouse represents a novel and useful mouse model to study PDB pathogenesis, and potentially, other pathologies associated with OPTN dysfunction, such as primary open-angle glaucoma (POAG) and amyotrophic lateral sclerosis (ALS).

While multiple studies have explored the molecular mechanisms by which OPTN dysfunction underlies disease pathogenesis, these studies have mainly focused on POAG and ALS [19]. Studies have demonstrated that the POAGassociated OPTN mutant (E50K) could selectively induce ROS-associated cell death of retinal ganglion cells in vitro [17], and OPTN E50K knock-in mice exhibit loss of retinal ganglion cells in vivo [36]. Furthermore, in response to TNF $\alpha$ signaling, OPTN deficiency leads to persistent RIPK1 signaling and uncontrolled necroinflammation in the spinal cord, resulting in axonal degeneration and ALS [18]. However, in contrast to the pathophysiology of Optn-dependent POAG and ALS, which is characterized by the loss of ganglion cells or neurons [18, 36], PDB is highlighted by a "gain" of osteoclasts [37], suggesting that the molecular mechanisms implicated in the pathogenesis of PDB is dissimilar to POAG and ALS. While Optn-deficient osteoclasts did not display any difference in RIPK1 expression at either mRNA and protein levels, it is possible that activation of

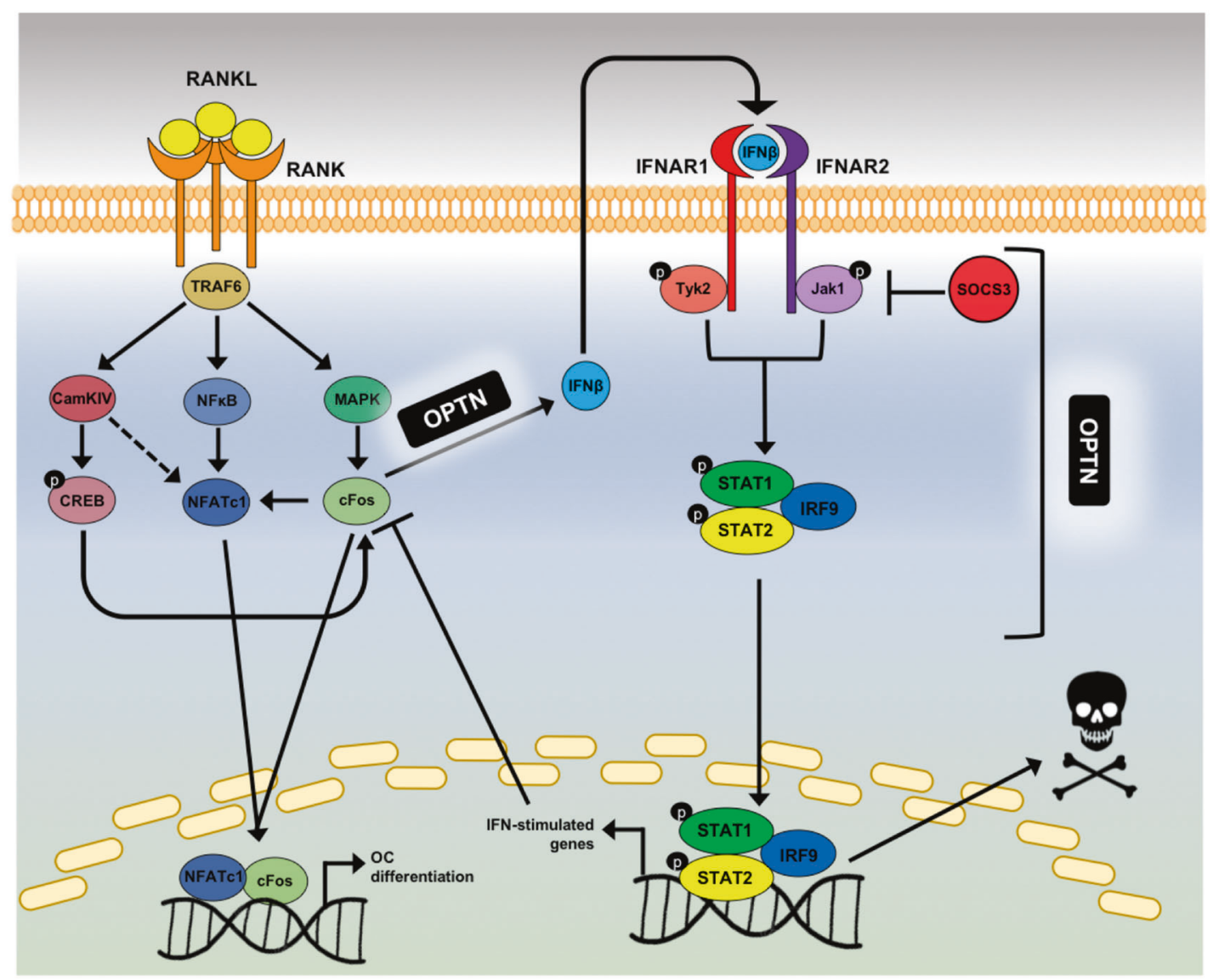

Fig. 6 Schematic of the dual role of OPTN in mediating negative regulation of osteoclastogenesis. During RANKL-mediated osteoclastogenesis, OPTN is required for optimal IFN $\beta$ production, which is critical for inhibition of $c-F o s$, promotion of the IFN $\alpha / \beta \mathrm{R}$ signaling cascade, and induction of cell death 
necroptotic machinery is altered in Optn-deficient osteoclasts. A recent study has identified MK2 has a critical negative regulator of RIPK1 activity, wherein MK2 phosphorylates RIPK1 at Ser321 to inhibit its ability to induce RIPK1dependent apoptosis and necroptosis [38]. Interestingly, $M K 2^{-/-}$mice display a significant decrease in osteoclastogenesis, which could suggest that an increase in functionally active RIPK1 results in decreased osteoclast survival [39]. The role that OPTN plays in modulating the MK2-RIPK1 axis in response to type I IFN remains to be elucidated.

Although OPTN has been shown to be a potent inhibitor of NF- $\kappa \mathrm{B}$ activity [11], our data showed that Optn-deficient osteoclasts do not have altered NF- $\mathrm{NB}$ activation ex vivo, consistent with recent reports [15, 16, 35]. However, failure to detect alterations in NF- $\mathrm{BB}$ activity in our study may be due to experimental limitations, as the previous study found increased NF- $\kappa \mathrm{B}$ responses in the Optn ${ }^{D 477 N / D 477 N}$ model using the $\mathrm{NF}-\kappa \mathrm{B}$ luciferase reporter assay [10], which may be more sensitive compared with western blotting. Indeed, we did observe a significant and two-pronged defect in the execution of the negative feedback loop mediated by IFN $\beta$. In the absence of OPTN, both production of IFN $\beta$ and signaling downstream of the IFN $\alpha / \beta \mathrm{R}$ are impaired. While previous studies have described a role for OPTN in IFN $\beta$ production during osteoclastogenesis [10], here we demonstrate for the first time that OPTN is also required for optimal signaling downstream of IFN $\beta$ engagement of the IFN $\alpha / \beta$ R (Fig. 6). This defect manifests itself in a failure to upregulate anti-osteoclastogenic factors, as well as promote cell survival. Thus, OPTN limits osteoclastogenesis at two distinct nodes, and is a critical factor in restricting uncontrolled bone resorption.

Moreover, a defect in type I IFN production could in fact account for the defect in IFN $\alpha / \beta$ R signaling, as type I IFN upregulates expression of key components of the IFN $\alpha / \beta R$ signaling pathway [40]. It is possible that Optn-deficient osteoclasts fail to produce amounts of type I IFN necessary to properly establish an IFN $\alpha / \beta \mathrm{R}$ signaling platform, as indicated by decreased levels of the total STAT1 and STAT2 in Optn-deficient precursors. In addition, supplementation with recombinant IFN $\beta$ rescues the hyperosteoclastogenic phenotype in Optn-deficient osteoclasts as it upregulates components of the IFN $\alpha / \beta \mathrm{R}$ signaling pathway, such as STAT1 and STAT2, as well as inhibit c-Fosmediated osteoclast differentiation. Furthermore, blocking IFN $\alpha / \beta \mathrm{R}$ signaling with anti-IFNAR Ab had a more profound effect on $\mathrm{Optn}^{+/+}$osteoclasts, suggesting that defects in IFN $\alpha / \beta \mathrm{R}$ signaling prevented antibody blockade from being fully effective in $O p t n^{-/-}$osteoclasts.

Interestingly, while Optn-deficient osteoclasts exhibit a defective type I IFN pathway, the measles virus, which elicits a robust type I IFN response, is strongly correlated to PDB development [41]. Indeed, this association could support why individuals harboring the $O P T N$ variant are prone to develop $\mathrm{PDB}$, as their intrinsic defects in the type I IFN pathway permit them to be more susceptible to measles virus infection. Because of Optineurin's dual function in maintaining bone homeostasis and antiviral type I IFN signaling, IFN $\beta$ could be a novel pharmacotherapy for PDB, as it provides restoration of dysregulated bone resorption in addition to antiviral functions.

Taken together, our results allow us to propose a possible mechanistic model of OPTN-associated PDB pathogenesis. At the molecular level, OPTN plays a dual role in the negative regulation of osteoclastogenesis-the production of type I IFN and signaling of the type I IFN through the IFN $\alpha / \beta \mathrm{R}$ to maintain bone homeostasis. Deficiency in OPTN fails to execute the proper inhibitory functions of type I IFN signaling, leading to hyperactivation of osteoclastogenesis and development of osteolytic lesions observed in PDB.

Acknowledgements We would like to thank Danielle Callaway of the University of Texas, Brendan Boyce of the University of Rochester, and Kim Mansky of University of Minnesota for their help on osteoclast primary culture. We thank the staff in Biomedical Research Imaging Center and Maya Styner at University of North Carolina for their imaging technical support. We also thank Kevin Gerrish and Rick Fannin in the Molecular Genomics Core at the National Institute of Environmental Health Sciences for microarray support. We also thank Michael Fessler (NIEHS), Donald N. Cook (NIEHS), Kevin Byrd (UNC School of Dentistry), and Richard Loeser (UNC School of Medicine) for their careful assistance with this manuscript. This work was supported in part by the NIH Intramural Research Program 1ZIAES10328601 (to J.M.), NIH/NIDCR R01DE022816 (to C.K.) and R90DE022527 (to SW.W.), Butler Pioneer Award (to H.T.), Alcon Research Institute (to H.T.), American Glaucoma Society (to H. T.), K08-EY021520 (to H.T.), and NEI core grant P30-EY005722 (to Duke Eye Center).

\section{Compliance with ethical standards}

Conflict of interest The authors declare that they have no conflict of interest.

Publisher's note: Springer Nature remains neutral with regard to jurisdictional claims in published maps and institutional affiliations.

\section{References}

1. Teramachi J, Nagata Y, Mohammad K, Inagaki Y, Ohata Y, Guise $\mathrm{T}$, et al. Measles virus nucleocapsid protein increases osteoblast differentiation in Paget's disease. J Clin Invest. 2016;126:1012-22.

2. Kukita A, Chenu C, McManus LM, Mundy GR, Roodman GD. Atypical multinucleated cells form in long-term marrow cultures from patients with Paget's disease. J Clin Invest. 1990;85:1280-6.

3. Rebel A, Malkani K, Basle M, Bregeon C. Osteoclast ultrastructure in Paget's disease. Calcif Tissue Res. 1976;2:187-99.

4. Menaa C, Reddy SV, Kurihara N, Maeda H, Anderson D, Cundy $\mathrm{T}$, et al. Enhanced RANK ligand expression and responsivity of 
bone marrow cells in Paget's disease of bone. J Clin Invest. 2000;105:1833-8.

5. Ralston SH, Albagha OM. Genetics of Paget's disease of bone. Curr Osteoporos Rep. 2014;12:263-71.

6. Albagha OM. Genetics of Paget's disease of bone. Bone Rep. 2015;4:756.

7. Morissette J, Laurin N, Brown JP. Sequestosome 1: mutation frequencies, haplotypes, and phenotypes in familial Paget's disease of bone. J Bone Min Res. 2006;21(Suppl 2):P38-44.

8. Sundaram K, Shanmugarajan S, Rao DS, Reddy SV. Mutant p62P392L stimulation of osteoclast differentiation in Paget's disease of bone. Endocrinology. 2011;152:4180-9.

9. Albagha OM, Visconti MR, Alonso N, Langston AL, Cundy T, Dargie R, et al. Genome-wide association study identifies variants at CSF1, OPTN and TNFRSF11A as genetic risk factors for Paget's disease of bone. Nat Genet. 2010;42:520-4.

10. Obaid R, Wani SE, Azfer A, Hurd T, Jones R, Cohen P, et al. Optineurin negatively regulates osteoclast differentiation by modulating NF-kappaB and interferon signaling: implications for Paget's disease. Cell Rep. 2015;13:1096-102.

11. Slowicka K, Vereecke L, van Loo G. Cellular functions of optineurin in health and disease. Trends Immunol. 2016;37:621-33.

12. Zhu G, Wu CJ, Zhao Y, Ashwell JD. Optineurin negatively regulates TNFalpha- induced NF-kappaB activation by competing with NEMO for ubiquitinated RIP. Curr Biol. 2007;17:1438-43.

13. Chibalina MV, Roberts RC, Arden SD, Kendrick-Jones J, Buss F. Rab8-optineurin-myosin VI: analysis of interactions and functions in the secretory pathway. Methods Enzymol. 2008;438:11-24.

14. Chibalina MV, Poliakov A, Kendrick-Jones J, Buss F. Myosin VI and optineurin are required for polarized EGFR delivery and directed migration. Traffic. 2010;11:1290-303.

15. Munitic I, Giardino Torchia ML, Meena NP, Zhu G, Li CC, Ashwell JD. Optineurin insufficiency impairs IRF3 but not NFkappaB activation in immune cells. J Immunol. 2013; 191:6231-40.

16. Meena NP, Zhu G, Mittelstadt PR, Giardino Torchia ML, Pourcelot M, Arnoult D, et al. The TBK1-binding domain of optineurin promotes type I interferon responses. FEBS Lett. 2016;590:1498-508.

17. Chalasani ML, Radha V, Gupta V, Agarwal N, Balasubramanian D, Swarup G. A glaucoma-associated mutant of optineurin selectively induces death of retinal ganglion cells which is inhibited by antioxidants. Invest Ophthalmol Vis Sci. 2007;48:1607-14.

18. Ito Y, Ofengeim D, Najafov A, Das S, Saberi S, Li Y, et al. RIPK1 mediates axonal degeneration by promoting inflammation and necroptosis in ALS. Science . 2016;353:603-8.

19. Toth RP, Atkin JD. Dysfunction of optineurin in amyotrophic lateral sclerosis and glaucoma. Front Immunol. 2018;9:1017.

20. Ma HL, Blanchet TJ, Peluso D, Hopkins B, Morris EA, Glasson SS. Osteoarthritis severity is sex dependent in a surgical mouse model. Osteoarthr Cartil. 2007;15:695-700.

21. Huang H, Skelly JD, Ayers DC, Song J. Age-dependent changes in the articular cartilage and subchondral bone of C57BL/6 mice after surgical destabilization of medial meniscus. Sci Rep. 2017;7:42294.

22. Xiu Y, Xu H, Zhao C, Li J, Morita Y, Yao Z, et al. Chloroquine reduces osteoclastogenesis in murine osteoporosis by preventing TRAF3 degradation. J Clin Invest. 2014;124:297-310.
23. Lee DJ, Tseng HC, Wong SW, Wang Z, Deng M, Ko CC. Dopaminergic effects on in vitro osteogenesis. Bone Res. 2015;3:15020.

24. Dillon CP, Weinlich R, Rodriguez DA, Cripps JG, Quarato G, Gurung P, et al. RIPK1 blocks early postnatal lethality mediated by caspase-8 and RIPK3. Cell . 2014;157:1189-202.

25. Seitz S, Priemel M, Zustin J, Beil FT, Semler J, Minne H, et al. Paget's disease of bone: histologic analysis of 754 patients. J Bone Min Res. 2009;24:62-9.

26. Dutta S, Sengupta P. Men and mice: relating their ages. Life Sci. 2016;152:244-8.

27. Al Nofal AA, Altayar O, BenKhadra K, Qasim Agha OQ, Asi N, Nabhan M, et al. Bone turnover markers in Paget's disease of the bone: a systematic review and meta-analysis. Osteoporos Int. 2015;26:1875-91.

28. Teramachi J, Zhou H, Subler MA, Kitagawa Y, Galson DL, Dempster DW, et al. Increased IL-6 expression in osteoclasts is necessary but not sufficient for the development of Paget's disease of bone. J Bone Min Res. 2014;29:1456-65.

29. Lin NY, Beyer C, Giessl A, Kireva T, Scholtysek C, Uderhardt S, et al. Autophagy regulates TNFalpha-mediated joint destruction in experimental arthritis. Ann Rheum Dis. 2013;72:761-8.

30. Abu-Amer Y. NF-kappaB signaling and bone resorption. Osteoporos Int. 2013;24:2377-86.

31. Krebs DL, Hilton DJ. SOCS: physiological suppressors of cytokine signaling. J Cell Sci. 2000;113(Pt 16):2813-9.

32. Lehtonen A, Matikainen S, Julkunen I. Interferons up-regulate STAT1, STAT2, and IRF family transcription factor gene expression in human peripheral blood mononuclear cells and macrophages. J Immunol. 1997;159:794-803.

33. Takayanagi H, Kim S, Matsuo K, Suzuki H, Suzuki T, Sato K, et al. RANKL maintains bone homeostasis through c-Fosdependent induction of interferon-beta. Nature . 2002; 416:744-9.

34. Hanada R, Hanada T, Sigl V, Schramek D, Penninger JM. RANKL/RANK-beyond bones. J Mol Med (Berl). 2011; 89:647-56

35. Slowicka K, Vereecke L, Mc Guire C, Sze M, Maelfait J, Kolpe A, et al. Optineurin deficiency in mice is associated with increased sensitivity to Salmonella but does not affect proinflammatory NFkappaB signaling. Eur J Immunol. 2016;46:971-80.

36. Tseng HC, Riday TT, McKee C, Braine CE, Bomze H, Barak I, et al. Visual impairment in an optineurin mouse model of primary open-angle glaucoma. Neurobiol Aging. 2015;36:2201-12.

37. Roodman GD, Windle JJ. Paget disease of bone. J Clin Invest. 2005; $115: 200-8$.

38. Jaco I, Annibaldi A, Lalaoui N, Wilson R, Tenev T, Laurien L, et al. MK2 phosphorylates RIPK1 to prevent TNF-induced cell death. Mol Cell. 2017;66:698-710. e5.

39. Herbert BA, Valerio MS, Gaestel M, Kirkwood KL. Sexual dimorphism in MAPK-activated protein kinase-2 (MK2) regulation of RANKL-induced osteoclastogenesis in osteoclast progenitor subpopulations. PLoS One. 2015;10:e0125387.

40. Ivashkiv LB, Donlin LT. Regulation of type I interferon responses. Nat Rev Immunol. 2014;14:36-49.

41. Kurihara N, Zhou H, Reddy SV, Garcia Palacios V, Subler MA, Dempster DW, et al. Expression of measles virus nucleocapsid protein in osteoclasts induces Paget's disease-like bone lesions in mice. J Bone Min Res. 2006;21:446-55. 